\title{
The differential adsorption of silanes from solution onto model E-glass surfaces using high resolution XPS
}

\author{
F.R.JONES*, J.L.THOMASON ${ }^{\dagger}$ and X.M.LIU \\ Department of Engineering Materials, University of Sheffield, Sheffield, S1 3JD, UK \\ ${ }^{\dagger}$ European Owens-Corning, Rue de Charneux, Battice, B-4651, Belgium
}

\begin{abstract}
$\gamma$-aminopropyltriethoxysilane (APS), $\gamma$-mercaptopropyltrimethoxysilane (MPS) and their mixture have been adsorbed onto acid-treated model E-glass fibres from aqueous solution with different concentrations. High resolution X-ray photoelectron spectroscopy (XPS) has been employed to characterize APS and MPS single silane coatings and the selective adsorption of APS/MPS mixed silane coating. It is found that the Si contribution from the silane can be distinguished from the Si contribution from the acid-treated E-glass fibres by fitting $\mathrm{Si}_{2 \mathrm{p} 1 / 2}$ and $\mathrm{Si}_{2 \mathrm{p} 3 / 2}$ peaks with components for $\mathrm{CSiO}_{3}$ and $\mathrm{SiO}_{4}$ environments. The adsorption isotherms of APS and MPS have been obtained by comparing the atomic concentrations of $\mathrm{N}, \mathrm{S}$ and $\mathrm{CSiO}_{3}$ groups. APS and MPS are equally adsorbed from 0.1\% APS/MPS mixed silane solution, however, MPS dominates the deposit on model E-glass fibres to a depth corresponding to the take-off-angle of $45^{\circ}$ when it is adsorbed from $0.5 \%$ and $1.0 \%$ APS/MPS mixed silane solutions.
\end{abstract}

Key words: E-glass fibres; model E-glass surfaces; silanes; coatings; adsorption; XPS. 


\section{INTRODUCTION}

Single and mixed organosilanes are often used in commercial sizing to provide increased compatibility of the fibres and promote the adhesion between glass fibre surfaces and the resin in a glass fibre reinforced polymer composite [1-3]. Mixed silanes can introduce different chemical properties to glass surfaces. This is fundamental to the optimisation of silane sizings for industrial applications. However, the use of mixed silanes may result in potential complications, since the additional reactions between the silanes can modify the selective adsorption process and change the efficiency of the adhesion.

Most X-ray photoelectron spectroscopy (XPS) studies have employed silanes with labeling element such as APS with nitrogen because the labeling element can be used to determine the extent of the interaction between silanes and glass surfaces [4-8]. However, some authors have used the difference of binding energy between $\mathrm{O} 1 \mathrm{~s}$ and Si2p to differentiate the silicon from the silane coating and from the glass substrate [9-11].

In this study, model experiments have been undertaken on nitric acid treated E-glass fibres by using high resolution XPS. $\gamma$-aminopropyltriethoxysilane (APS) and $\gamma$ mercaptopropyltrimethoxysilane (MPS) were chosen because the nitrogen and sulphur provide labeling elements. To separate the siloxane contribution from that of the model glass substrate and to assess the preferential adsorption from APS/MPS mixed silane solution were the focuses of this study.

\section{EXPERIMENTAL}

\subsection{Materials}

To produce a silica-like surface, model E-glass fibres were prepared by immersing Eglass fibres (Owens-Corning) in concentrated nitric acid (69\%, analytical reagent, Aldrich) for 6 days at room temperature, then washed twice in fresh nitric acid and deionised water. In this case $\mathrm{Al}, \mathrm{Ca}$ and $\mathrm{Mg}$ are extracted leaving a surface composed of mainly Si-O and Si-OH. 
$\gamma$-aminopropyltriethoxysilane (APS), $\gamma$-mercaptopropyltrimethoxysilane (MPS) and methanol described in Table 1, were used as received without further purification.

Table 1.

Silanes used in silanisation

\begin{tabular}{ccc}
\hline Silane & Chemical Structure & Company \\
\hline APS & $\mathrm{NH}_{2}-\left(\mathrm{CH}_{2}\right)_{3}-\mathrm{Si}\left(\mathrm{OC}_{2} \mathrm{H}_{5}\right)_{3}$ & GE Speciality Materials \\
MPS & $\mathrm{HS}-\left(\mathrm{CH}_{2}\right)_{3}-\mathrm{Si}\left(\mathrm{OCH}_{3}\right)_{3}$ & Sigma-Aldrich \\
Methanol & $\mathrm{CH}_{3}-\mathrm{CH}_{2}-\mathrm{OH}$ & Sigma-Aldrich \\
\hline
\end{tabular}

\subsection{Silane treatments}

Different concentrations $(0.1 \%, 0.5 \%$ and $1.0 \%)$ and compositions (APS, MPS and APS/MPS mixture) of silane solutions were prepared. For single silane, APS was hydrolytically dissolved in deionised water. MPS was dissolved in a mixture of methylated spirit $(20 \%)$ and deionised water $(80 \%)$. Mixed silane solution was prepared by mixing the same concentration APS and MPS single silane solutions.

The model E-glass fibres (after nitric acid treatment) were respectively immersed in the solution of APS, MPS and APS/MPS (1:1) for 15 minutes at room temperature. Then they were washed with fresh deionised water for three times and dried in a vacuum oven at $50^{\circ} \mathrm{C}$ for 24 hours [12].

\subsection{XPS analysis}

High resolution XPS was carried out using the SCIENTA ESCA 300 spectrometer operated in the constant analyzer energy mode, at the pass energy of $40 \mathrm{eV}$. The monochromatic Al Ka X-ray source $(\eta \nu=1486.7 \mathrm{eV})$ was used and the take-off-angle was set at $45^{\circ}$. The electron analyzer energy slit width was set at $1.9 \mathrm{~mm}$ during wide scans and at $0.8 \mathrm{~mm}$ for the narrow scans. Charge compensation was achieved using an electron flood gun of $4-6 \mathrm{eV}$. The instrument operating pressure during analysis was less than $10^{-8}$ Torr.

$\mathrm{O} 1 \mathrm{~s}(533.0 \mathrm{eV})$ was employed as a reference for the calculation of the binding energy. 
The relative concentrations of all the elements, present in the wide scans, were calculated using the atomic sensitivity factors specific to the SCIENTA instrument. Curve-fitting after background subtraction was conducted assuming a complex mixed Gaussian-Lorentzian peak shape. Asymmetry was implemented with a tail function dependent on the photoelectron energy $[13,14]$.

\section{RESULTS AND DISCUSSION}

\subsection{MPS coated model E-glass fibres}

The relative surface compositions of the untreated and model E-glass fibres after nitric acid treatment are given in Table 2. No Al and $\mathrm{Ca}$ are signals detected in the survey scan of nitric acid treated model E-glass fibres at a take-off-angle of $45^{\circ}$. Thus, model E-glass surfaces with only $\mathrm{Si}, \mathrm{O}$ and $\mathrm{C}$ are prepared. According to previous unpublished angle resolved XPS study, the C intensity decreased with the increase of take-off-angle, indicating the $\mathrm{C}$ on the surface is from the contamination.

Wide scan spectrum and narrow scan spectrum in region S2p of $1 \%$ MPS coated model E-glass fibres are shown in Figure 1. Deposition of the MPS is confirmed by the introduction of the S2s and S2p peaks at 225 and $164 \mathrm{eV}$. S2p spectrum (Figure $1 \mathrm{~b}$ ) is fitted with a doublet at 163.7 and $164.9 \mathrm{eV}$, which can be attributed to S-C or S-H bonds.

The atomic concentrations of model E-glass surfaces with different concentration MPS coatings have been calculated as described above and are shown in Table 2. The Si concentration is shown to be reduced on deposition of silane because Si in the MPS is lower than that in model E-glass fibres. On increasing the concentration of MPS, a thicker coating was achieved, which can be shown by the increase in $\mathrm{S}$ and $\mathrm{C}$ intensities and the reduction in Si intensity. Compared to the same concentration APS deposits (Table 2), higher C intensities and lower Si intensities were detected for MPS, which shows that the MPS coating has a higher thickness than that from APS. This is because the MPS deposit was deposit from a solution in alcohol and deionised water, whereas the APS deposit was prepared from deionised water alone. The increased thickness can be attributed to differing degrees of hydrolysis and polymerization. 
Table 2.

The atomic concentrations of untreated and acid-treated E-glass fibres determined from the survey scan at a take-off-angle of $45^{\circ}$.

\begin{tabular}{|c|c|c|c|c|c|c|c|c|}
\hline & Silane $(\%)$ & $\mathrm{O}$ & $\mathrm{C}$ & $\mathrm{Si}$ & $\mathrm{S}$ & $\mathrm{N}$ & $\mathrm{Ca}$ & $\mathrm{Al}$ \\
\hline Untreated E-glass & -- & 57.0 & 16.9 & 15.8 & -- & -- & 4.5 & 5.8 \\
\hline Model E-glass & -- & 61.6 & 10.8 & 27.6 & -- & -- & -- & -- \\
\hline \multirow[t]{3}{*}{ APS coated model E-glass } & 0.1 & 57.2 & 14.0 & 28.0 & -- & 0.8 & -- & -- \\
\hline & 0.5 & 56.1 & 18.1 & 24.0 & -- & 1.8 & -- & -- \\
\hline & 1.0 & 57.1 & 21.2 & 19.5 & -- & 2.2 & -- & -- \\
\hline \multirow[t]{3}{*}{ MPS coated model E-glass } & 0.1 & 55.4 & 17.2 & 25.5 & 1.7 & -- & -- & -- \\
\hline & 0.5 & 49.5 & 27.5 & 18.9 & 4.1 & -- & -- & -- \\
\hline & 1.0 & 47.0 & 30.0 & 17.5 & 5.5 & -- & -- & -- \\
\hline \multirow{3}{*}{$\begin{array}{l}\text { APS/MPS coated model E- } \\
\text { glass }\end{array}$} & 0.1 & 63.4 & 13.2 & 22.3 & 0.6 & 0.5 & -- & -- \\
\hline & 0.5 & 34.9 & 38.4 & 20.2 & 4.1 & 2.4 & -- & -- \\
\hline & 1.0 & 26.2 & 46.1 & 16.6 & 8.5 & 2.6 & -- & -- \\
\hline
\end{tabular}

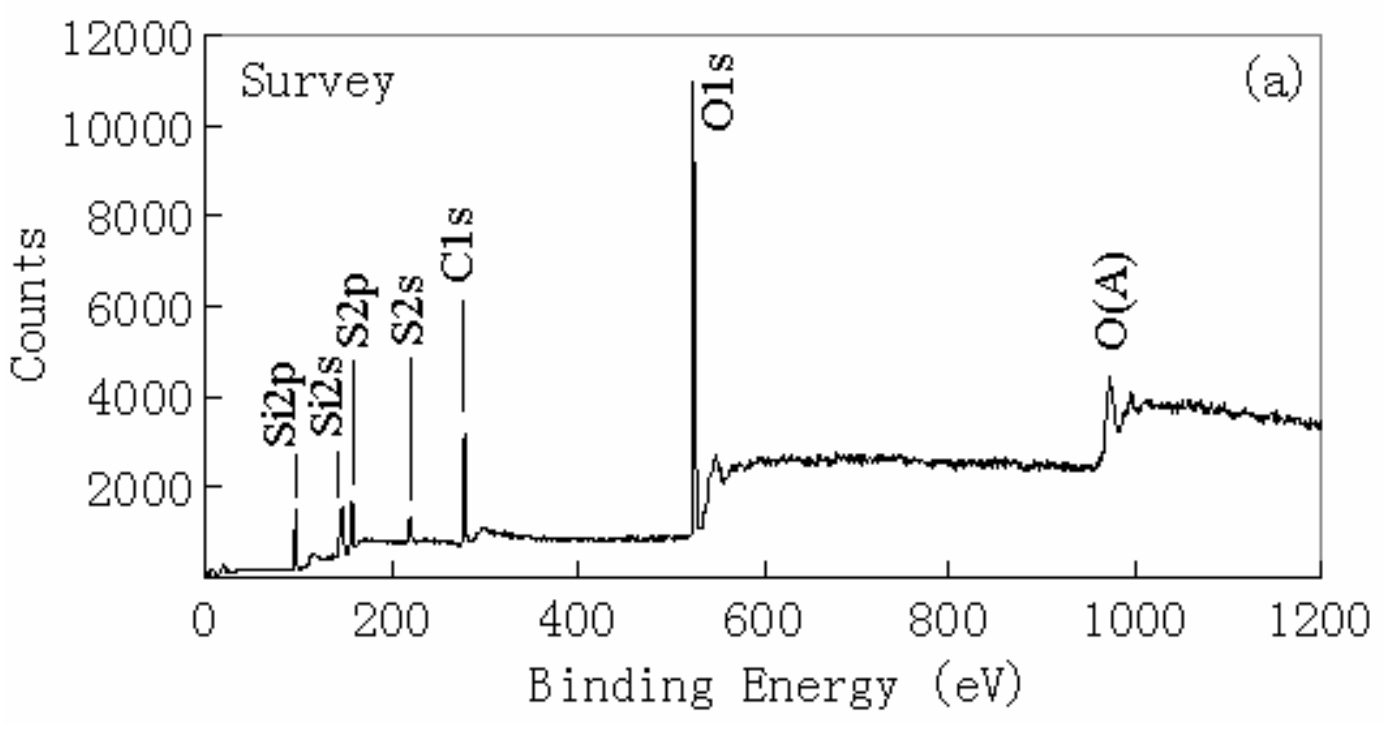




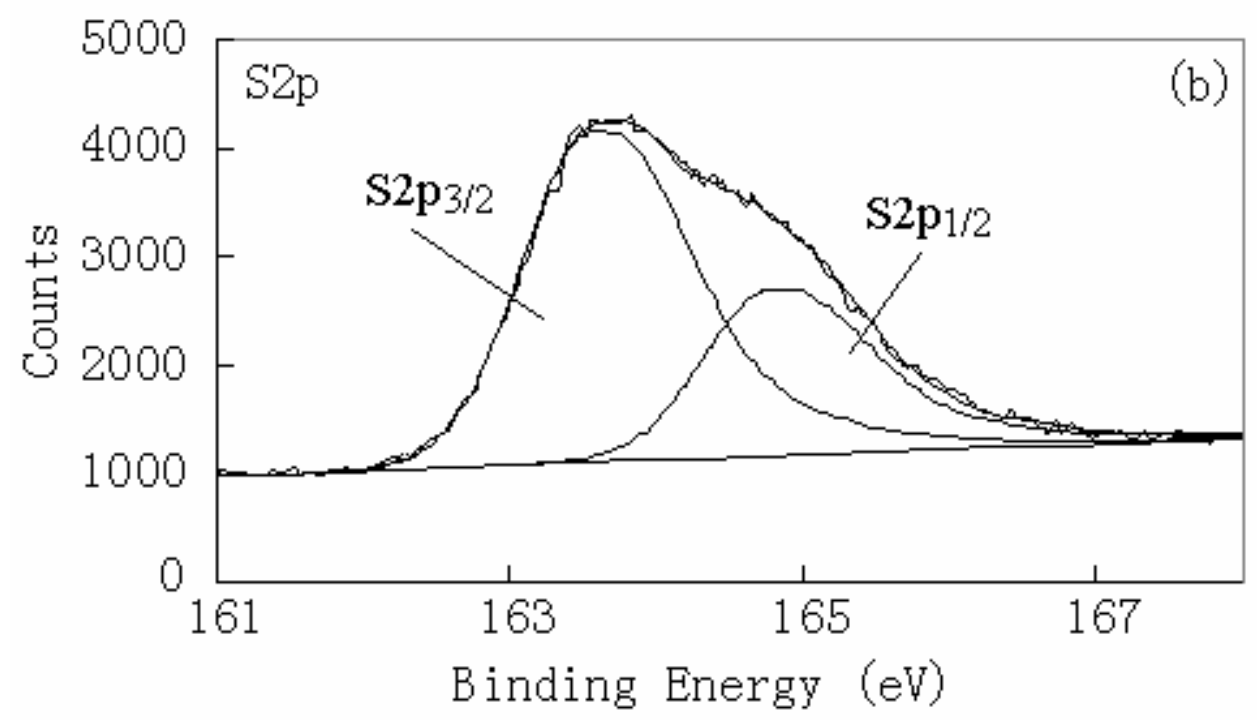

Figure 1. XPS spectra of 1\% MPS coated model E-glass fibres: (a) survey scan and (b) $\mathrm{S}_{2 \mathrm{p}}$.

The Si2p spectra for untreated E-glass surface, model E-glass surface after nitric acid treatment and 0.1, 0.5 and 1\% MPS coated model E-glass surface are shown in Figure 2. The $\mathrm{Si} 2 \mathrm{p}_{3 / 2}$ and $\mathrm{Si} 2 \mathrm{p}_{1 / 2}$ peaks of the model E-glass surface from $\mathrm{Si}-\mathrm{O}$ and $\mathrm{Si}-\mathrm{OH}$ appears at the binding energy of $103.5 \mathrm{eV}$ and $104.2 \mathrm{eV}$ with a FWHM of $\sim 1.5 \mathrm{eV}$ (Figure 2b). When the model surface is coated with MPS, the Si2p peak (Figure 2c, $2 \mathrm{~d}$ and $2 \mathrm{e}$ ) broadened because of the introduction of an extra doublet peak from $\mathrm{CSiO}_{3}$ at $102.4 \mathrm{eV}$ and $103.1 \mathrm{eV}$. Since the chemical shift in binding energy is approximately $1.1 \mathrm{eV}$, the contribution from the silane deposit $\left(\mathrm{CSiO}_{3}\right)$ can be differentiated from that of the model E-glass substrate $\left(\mathrm{SiO}_{4}\right)$. But for untreated Eglass surface, the $\mathrm{Si} 2 \mathrm{p}_{3 / 2}$ appears at $102.4 \mathrm{eV}$ (Figure 2a), which is identical to the binding energy expected from the $\mathrm{Si} 2 \mathrm{p} 3 / 2$ line of the silane deposit. So it is impossible to separate Si contributions from the silane and E-glass substrate since both appear at the same binding energy.

As seen in Figure 2 (c), (d) and (e), the percentage of $\mathrm{CSiO} 3$ component increased with the increase of concentration of the MPS solution, which means that a higher proportion of detectable $\mathrm{Si}$ arises from the silane deposit adsorbed onto model E-glass surface. In other words, a thicker coating is formed on 1\% MPS coated model E-glass surface. This is consistent with the atomic concentration results of MPS coated surfaces mentioned above. The $\mathrm{S}$ and $\mathrm{C}$ intensities increase and the Si intensity 
decreases with the increase of MPS concentration.
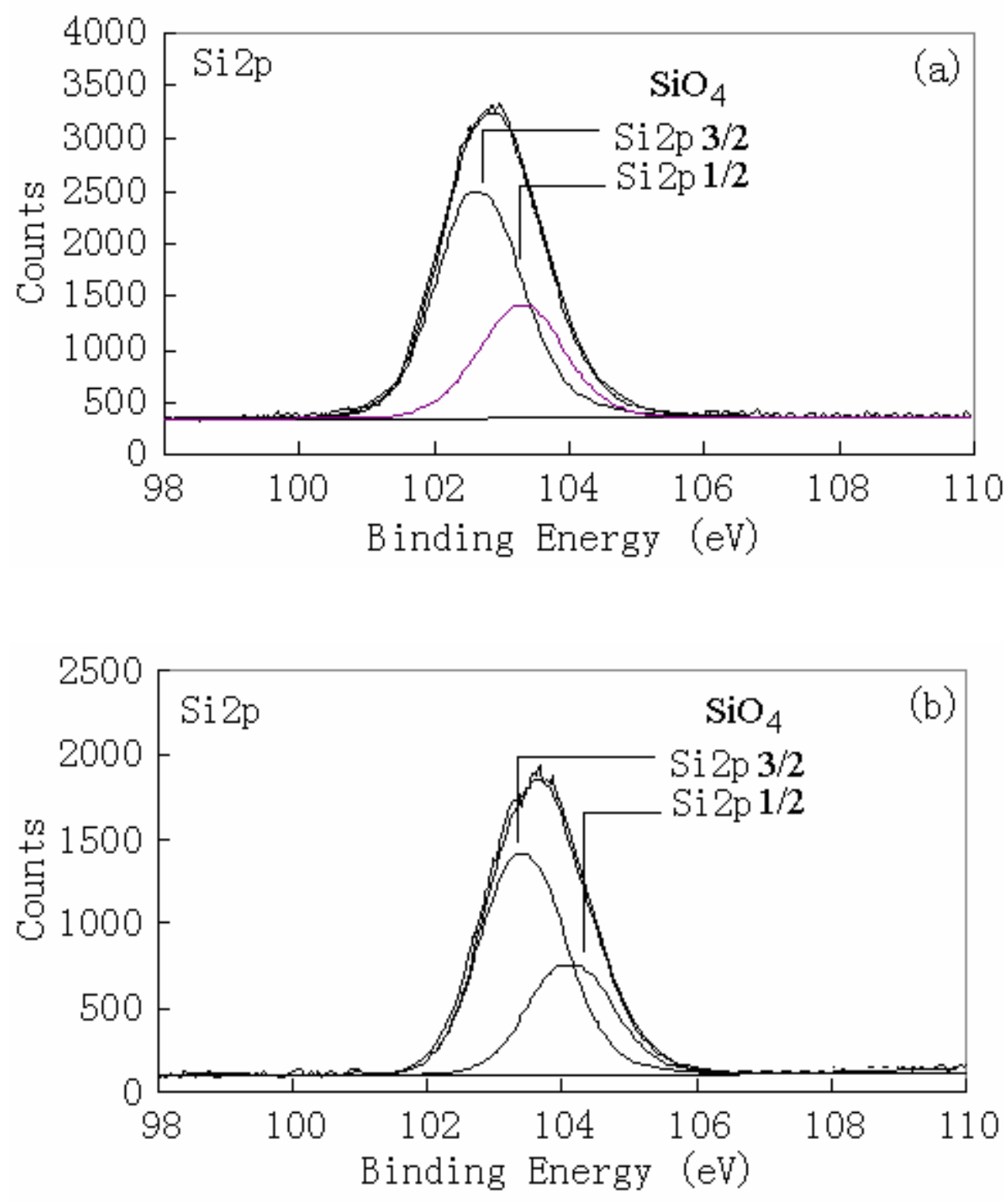

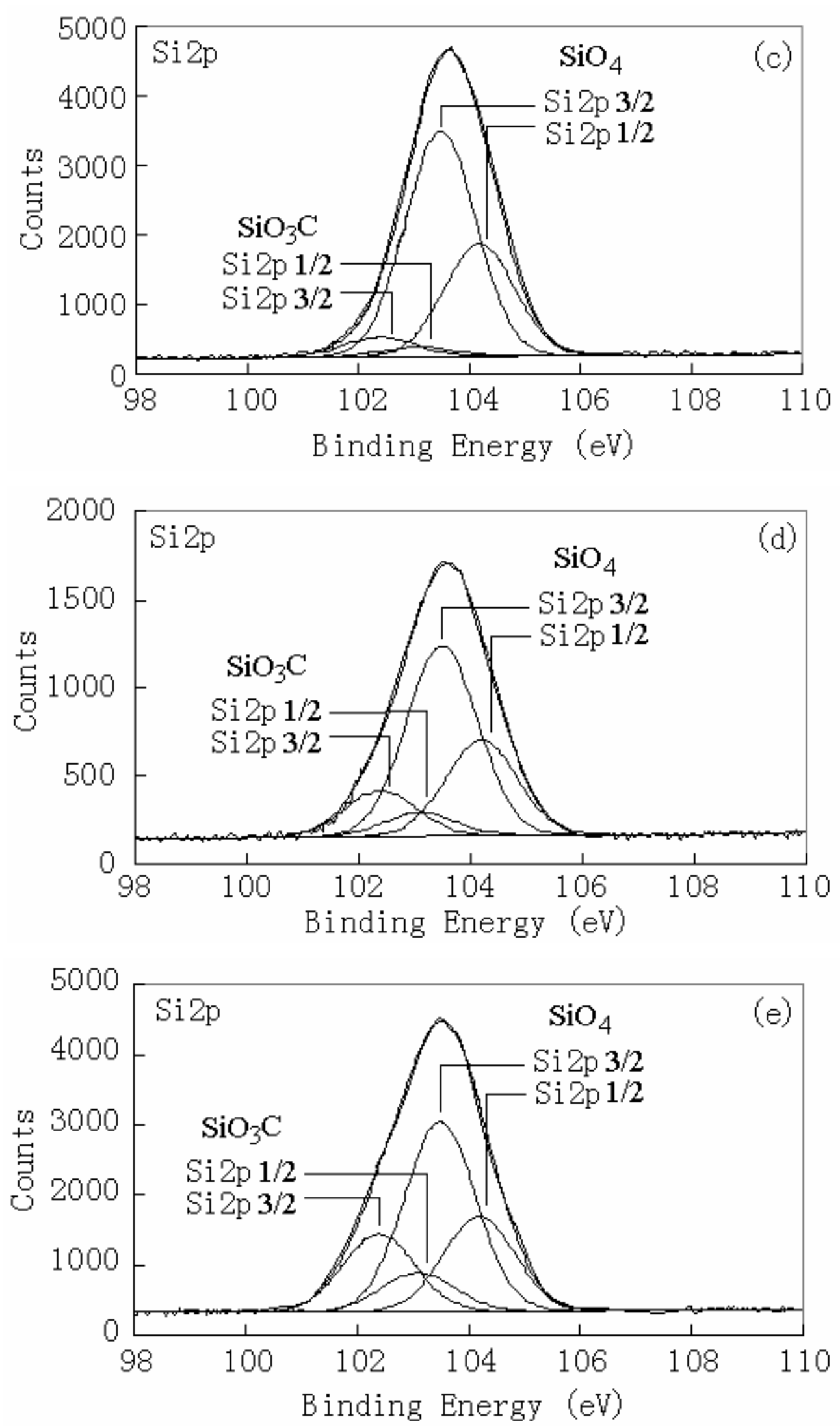

Figure 2. Si2p XPS spectra from the (a) untreated E-glass surface, (b) model E-glass surface, (c) 0.1\% MPS coated model E-glass surface, (d) 0.5\% MPS coated model Eglass surface and (e) 1.0\% MPS coated model E-glass surface. 


\subsection{MPS/APS-mixed-silane-coated model E-glass fibres}

As shown in Table 2 and Figure 3, S and N signals are detected on MPS/APS mixed silane coated model E-glass surfaces, indicating that both MPS and APS are involved in the silane deposit. For the 1\% APS/MPS coating, the atomic concentrations for O, $\mathrm{C}, \mathrm{Si}, \mathrm{S}$ and $\mathrm{N}$ are $26.2,46.1,16.6,8.5$ and $2.6 \%$ respectively. There is more $\mathrm{C}, \mathrm{S}$ and $\mathrm{N}$ and less $\mathrm{O}$ and $\mathrm{Si}$ in the surface with an APS/MPS mixed-silane deposit compared to individually deposited APS and MPS. This can be attributed to the thicker mixed deposit, which is confirmed by the curve fit of Si2p (Figure $3 b$ ). $62 \%$ of Si is from the APS/MPS mixed silane deposit $\left(\mathrm{C} \mathrm{SiO}_{3}\right)$, which is much higher than $29 \%$ for MPS and $12 \%$ for APS.
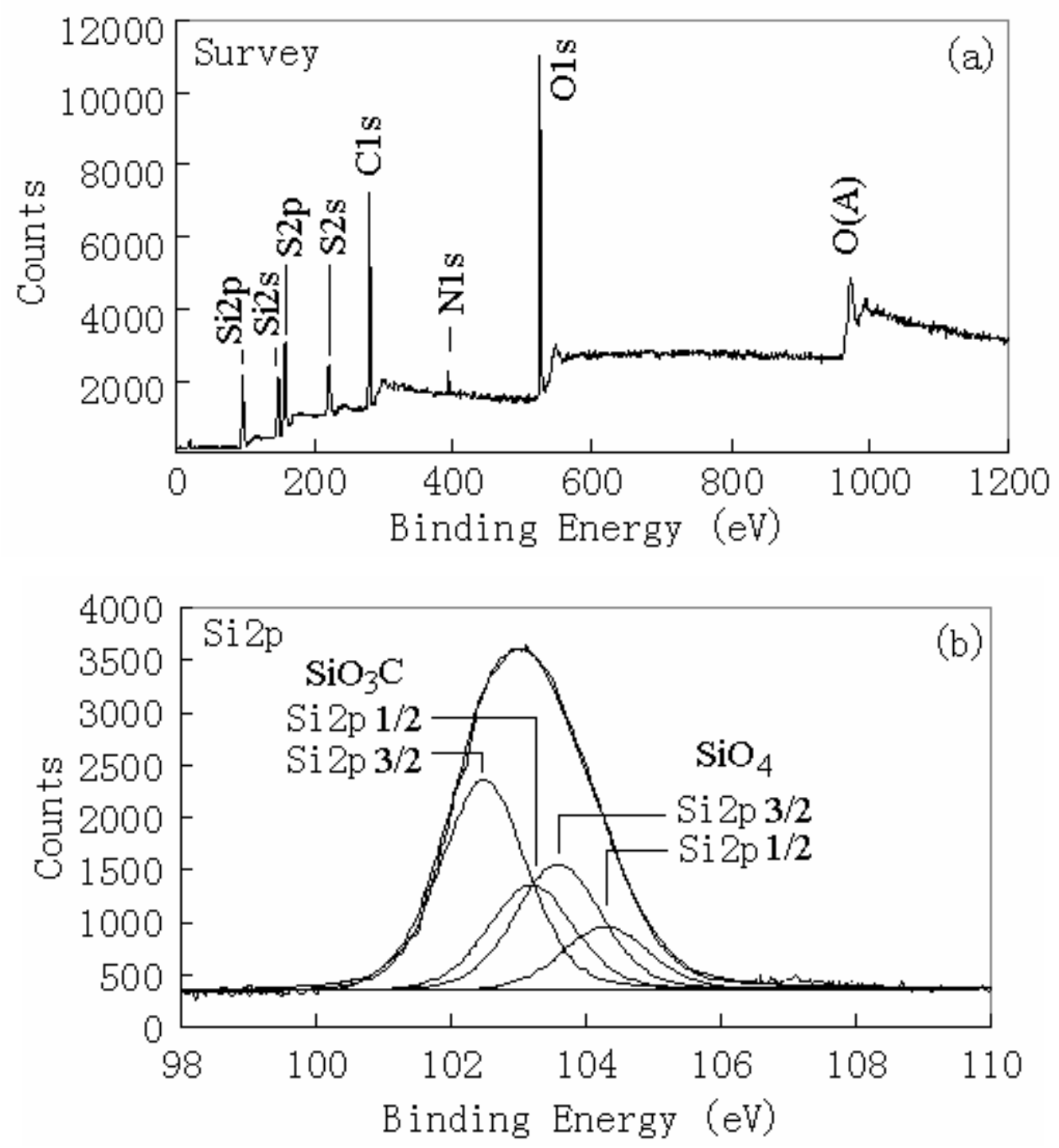

Figure 3. XPS spectra of 1\% MPS/APS-mixed-silane-coated model E-glass fibres: (a) survey scan and (b) Si2p. 
The adsorption isotherms for APS/MPS mixed silanes on model E-glass surfaces are shown in Figure 4. The $\mathrm{S}$ and $\mathrm{N}$ atomic concentrations obtained from wide scan spectra and siloxane contribution from the Si2p curve fitting have been plotted against the concentration of the APS/MPS mixed silane solution. Since $1 \mathrm{~mol}$ APS has $1 \mathrm{~mol}$ - $\mathrm{NH}_{2}$ groups and $1 \mathrm{~mol}$ MPS has $1 \mathrm{~mol}-\mathrm{SH}$ groups (Table 1), the percentage of APS and MPS involved in the mixed silane deposit can be obtained from the atomic concentrations of N and S. According to the chemical structures of APS and MPS in Table 1, $1 \mathrm{~mol} \mathrm{CSiO}_{3}$ group is present in each silane, which can also give the amount of silane involved in the deposit. In Figure 4 the $\mathrm{CSiO}_{3}$ intensity shown by the continuous line with rhombic dots is obtained from Si2p curve fitting. The sum atomic concentration of $\mathrm{S}$ and $\mathrm{N}$ is represented by the dashed line. Basically, they show the similar trend, which confirms that it is possible to determine the degree of adsorption of silane onto model E-glass surfaces by the differentiation between $\mathrm{CSiO}_{3}$ and $\mathrm{SiO}_{4}$.

At $0.1 \%$ mixed silane concentration, MPS and APS are absorbed onto model E-glass surface at 1:1 ratio, which is similar to the initial ratio of MPS to APS input in the solution. But MPS to APS ratios are 1.7 and 3.3 respectively when the silane concentration increases to $0.5 \%$ and $1.0 \%$. There are two possibilities to account for this observation. One is that the siloxane deposit on the model E-glass fibres is homogeneous with a higher MPS concentration than APS. The other possibility is that APS is adsorbed preferentially over MPS onto the model E-glass fibre surface, so that as the concentration of MPS in the solution increases, it will be deposited on top of the initial APS layer. Thus the APS:MPS ratio in the deposit will vary with solution concentration. 


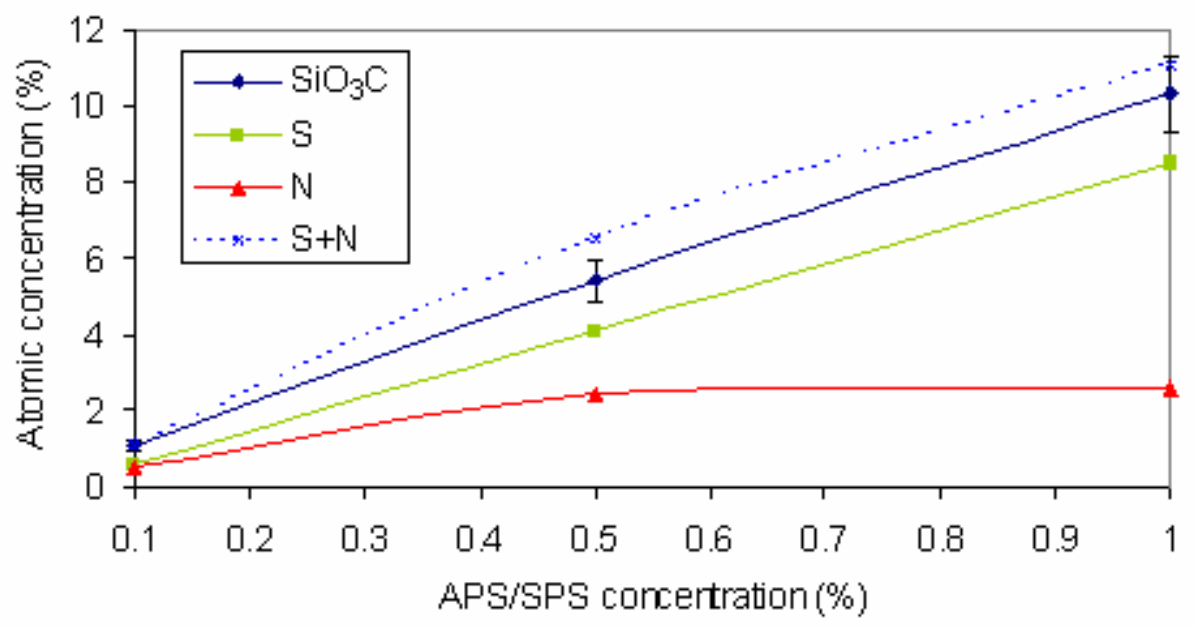

Figure 4. XPS results for labelling elements $S$ and $N$ concentrations from wide scan spectra and siloxane contribution from mixed-silane deposit $\left(\mathrm{CSiO}_{3}\right)$ obtained from Si2p curve fitting versus APS/MPS mixed-silane concentration.

\section{CONCLUSIONS}

Model E-glass surfaces without $\mathrm{Al}$ and $\mathrm{Ca}$ have been prepared with nitric acid treatment. In the XPS spectrum, the $\mathrm{Si} 2 \mathrm{p}_{3 / 2}$ and $\mathrm{Si} 2 \mathrm{p}_{1 / 2}$ peaks from the model E-glass $\left(\mathrm{SiO}_{4}\right)$ appear at $103.5 \mathrm{eV}$ and $104.2 \mathrm{eV}$ respectively. After MPS treatment, the silanised model E-glass has a broad Si2p peak in the XPS spectrum, which can be fitted with components for $\mathrm{SiCO}_{3}$ at $102.4 \mathrm{eV}$ and at $103.1 \mathrm{eV}$. Therefore, from the XPS spectrum, the Si contribution from the silane can be distinguished from the model E-glass substrate. This enables the adsorption isotherms for APS and MPS on model E-glass surfaces to be obtained. Especially for mixed silanes, APS and MPS are adsorbed onto model E-glass at 1:1, 1:1.7 and 1:3.3 ratios from APS/MPS mixed silane solutions of $0.1 \%, 0.5 \%$ and $1.0 \%$ respectively.

\section{ACKNOWLEDGEMENT}

The authors would like to thank Owens Corning for the financial support. We are also grateful to EPSRC for the access to ESCA at Daresbury Laboratory. 


\section{REFERENCES}

[1] Ishida, H, Adhesion Aspects of Polymeric Coatings, Plenum Press, New York, 1983.

[2] Plueddeman E P, Silane Coupling Agents, Plenum Press, New York, 1982.

[3] Mittal K L, Silane and Other Coupling Agents, Utrecht: VSP, 1992.

[4] Ishida H, Naviroj S, Tripathy S, Fitzgerald J J and Koenig J L, J. Polym. Sci., Polym. Phys. Ed., 20 (1992) 701.

[5] Chiang C H, Ishida H and Koenig J L, J. Coll. Interface Sci., 74 (1980) 396.

[6] Wang D, Jones F R and Denison P, J. Adhesion Sci. and Tech., 6 (1992) 79.

[7] Wang D and Jones F R,, J. Mater. Sci., 28 (1993) 2481.

[8] Abel M L, Watts J F and Digby R P, International Journal of Adhesion and Adhesives, 18 (1998) 179.

[9] Wagner C D, Passoja D E, Hillery H F, Kinisky T G, Six H A, Jansen W T and Taylor J A, J. Vac. Sci. Technol., 21 (1982) 993.

[10] Watson H, Mikkola P J, Matisons J G and Rosenholm J B, Colloids and Surfaces A: Physicochemical and Engineering Aspects, 161 (2000) 183.

[11] Watson H, Mikkola P J, Rosenholm J B, Matisons J G, Colloid Polym. Sci., 279 (2001) 1020 .

[12] T. Choudhury and F.R. Jones, International Journal of Adhesion and Adhesives, $26(2006) 79$.

[13] Beamson G and Briggs D, "High resolution XPS of Organic Polymers", The Scienta ESCA300 Database, John Wiley \& Sons, 1992.

[14] Briggs D and Seah M P, Practical Surface Analysis, $2^{\text {nd }} e d$. Vol. 1 - Auger and Xray Photoelectron Spectroscohomapy, New York, 1995 